\title{
Uncertainty Measures and Prediction Quality Rating for the Semantic Segmentation of Nested Multi Resolution Street Scene Images
}

\author{
Matthias Rottmann ${ }^{1}$ and Marius Schubert $^{1}$ \\ ${ }^{1}$ University of Wuppertal, School of Mathematics and Natural Sciences \\ \{rottmann, marius.schubert\} @uni-wuppertal.de
}

\begin{abstract}
In the semantic segmentation of street scenes the reliability of the prediction and therefore uncertainty measures are of highest interest. We present a method that generates for each input image a hierarchy of nested crops around the image center and presents these, all re-scaled to the same size, to a neural network for semantic segmentation. The resulting softmax outputs are then post processed such that we can investigate mean and variance over all image crops as well as mean and variance of uncertainty heat maps obtained from pixel-wise uncertainty measures, like the entropy, applied to each crop's softmax output. In our tests, we use the publicly available DeepLabv3+ MobilenetV2 network (trained on the Cityscapes dataset) and demonstrate that the incorporation of crops improves the quality of the prediction and that we obtain more reliable uncertainty measures. These are then aggregated over predicted segments for either classifying between Io $U=0$ and IoU $>0$ (meta classification) or predicting the IoU via linear regression (meta regression). The latter yields reliable performance estimates for segmentation networks, in particular useful in the absence of ground truth. For the task of meta classification we obtain a classification accuracy of $81.93 \%$ and an AUROC of $89.89 \%$. For meta regression we obtain an $R^{2}$ value of $84.77 \%$. These results yield significant improvements compared to other approaches.
\end{abstract}

\section{Introduction}

In recent years, deep learning has outperformed other classes of predictive models in many applications. In some of these, e.g. autonomous driving or diagnostic medicine, the reliability of a prediction is of highest interest. In classification tasks, thresholding on the highest softmax probability or thresholding on the entropy of the classification distributions (softmax output) are commonly used approaches to detect false predictions of neural networks, see e.g. [9, 14]. Metrics like classification entropy or the highest softmax probability are also combined with model uncertainty (Monte-Carlo (MC) dropout inference) or input uncertainty, cf. [7] and [14], respectively. See [15] for further uncertainty metrics. These approaches have proven to be practically efficient for detecting uncertainty and some of them have also been transferred to semantic segmentation tasks. The work presented in [13] makes use of MC dropout to model the uncertainty of segmentation networks and also shows performance improvements in terms of segmentation accuracy. This approach was used in other works to model the uncertainty and filter out predictions with low reliability, cf. e.g. [12, 19]. In [10] this line of research was further developed to detect spacial and temporal uncertainty in the semantic segmentation of videos. In [16] the concept of meta classification in semantic segmentation, the task of predicting whether a predicted segment intersects with the ground truth or not, was introduced. This can be formulated as the task of classifying between $I o U=0$ and $I o U>0$ for every predicted segment (the $I o U$ is also known as Jaccard index [11]). Furthermore a framework for the prediction of the $I o U$ via linear regression (meta regression) was proposed. The prediction of the $I o U$ can be seen as a performance estimate which, after training a model, can be computed in the absence of ground truth. Both predictors use segment-wise metrics extracted from the segmentation network's softmax output as its input. A visualization of a segment-wise $I o U$ rating is given in fig. 1. Apart from the discussed uncertainty related methods, there are also works based on input image statistics. For instance, in [8] a method for the rejection of false positive predictions is introduced. Performance measures for the segmentation of videos, also based on image statistics and boundary shapes, is introduced in [6].

In this work we elaborate on the uncertainty based approach from [16] which is a method that consists of three simple steps. First, the segmentation network's softmax output is used to generate uncertainty heat maps, e.g. the pixel-wise entropy (cf. fig. 3). In the second step, these un- 


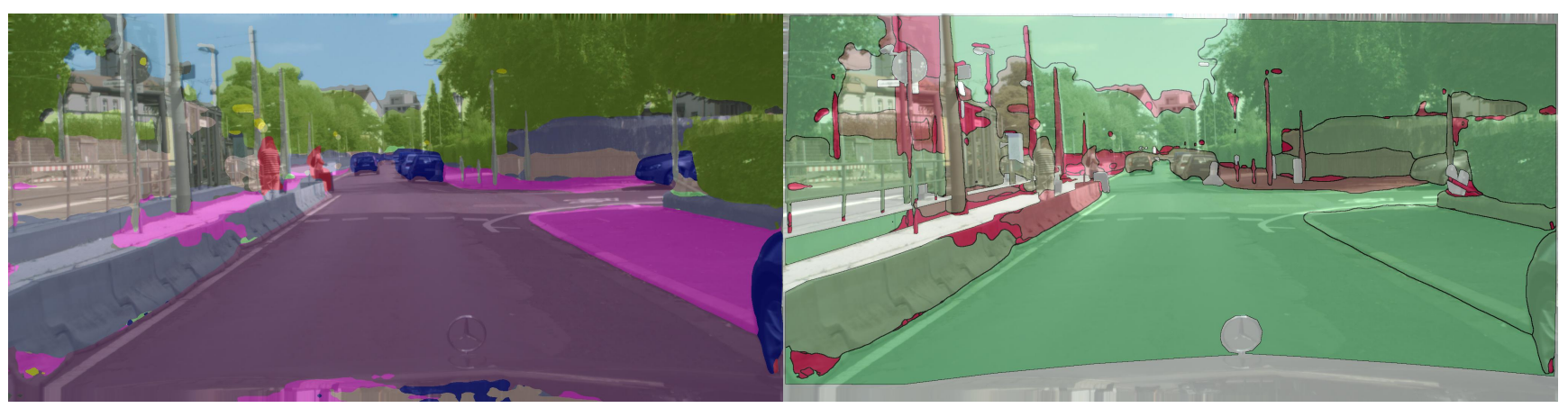

Figure 1. (Left): segmentation predicted by a neural network, (right): a visualization of the $I o U$ which can only be computed in the presence of ground truth. Green color corresponds to high $I o U$ values and red color to low ones, for the white regions there is no ground truth available. These regions are excluded from statistical evaluations.

certainty heat maps are then aggregated over the predicted segments and combined with other quantities derived from the predicted segments, e.g. the number of pixels per segment. From this we obtain segment-wise metrics. In the third step, these metrics are inputs for either a meta classification (between $I o U=0$ and $I o U>0$ ) or a meta regression for predicting the $I o U$. In this paper, we perform the same prediction tasks, however we improve the method in all of its three steps.

In many scenarios, the camera system in use provides images with very high-resolution which are coarsened before presenting them to the segmentation network. Thus we loose information, especially for objects further away from the camera. Therefore we propose a method that constructs a hierarchy of nested image crops where all images have a common center point, see fig. 2. All crops are then resized to the input size expected by the segmentation network such that we obtain an equally sized batch of input images. This can be processed by the neural network in a data parallel batch mode. Most neural network libraries, like e.g. Tensorflow [1], are well vectorized over the input batch. Thus the increase in execution time should be below linear. The outputs of the segmentation network are then scaled back to its original size. In addition, we add kernel functions to let the crops smoothly fade into the combination of all larger crops, that have been merged with their predecessors recursively in the same way. We do this in order to avoid boundary effects. From this procedure we obtain a batch of probability distributions that are inputs to uncertainty measures, e.g. the entropy, probability margin and variation ratio. These are applied pixel-wise and yield heat maps for each probability distribution. A mean and a variance over all image crop heat maps give us additional uncertainty information compared to the uncertainty information used in [16].

Furthermore we elaborate on the approach from [16] by introducing additional metrics that are derived from each segment's uncertainty and geometry information. In sum- mary we end up with 42 metrics (plus 19 predicted class probabilities averaged over the predicted segments) in contrast to the 15 metrics (plus 19 class probabilities) introduced in [16]. In addition to that, we study the incorporation of neural networks in meta classification and regression.

In our tests, we employ the publicly available DeepLabv3 + MobilenetV2 network [3, 17] that was trained on the Cityscapes dataset [4]. We perform all tests on the Cityscapes validation set. We demonstrate that the mean probability distribution over all crops provides improved $I o U$ values and that the additional uncertainty heat maps, respectively their mean and variance, yield improved uncertainty information which results in better inputs for meta classification and regression. For the task of meta classification we obtain a classification accuracy of $81.93 \%$ and an AUROC of $89.89 \%$. For meta regression we obtain an $R^{2}$ value of $84.77 \%$. We also show that these results yield significant improvements compared to baseline approaches and the results obtained by the predecessor method introduced in [16].

The remainder of this work is structured as follows: In section 2 we introduce the construction of the nested image crops, the aggregation of their softmax outputs and the resulting uncertainty heat maps. This is followed by the construction of segment-wise metrics using uncertainty and geometry information in section 3 . Afterwards we present numerical results. First, we study the segmentation performance for different numbers of image crops. Then, we study how useful our segment-wise metrics are for meta classification and regression. This also includes a variable/metric selection study. Afterwards, we compare the meta classification and regression performance of our approach with baseline approaches and previous ones. Lastly, we study the incorporation of neural networks in meta classification and regression. 


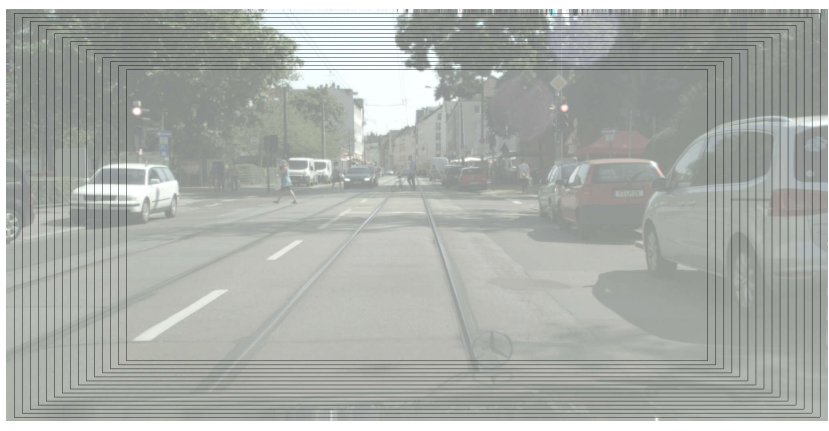

Figure 2. Visualization of a nested image cropping where all crops have the image center as focal point. This image is part of the Cityscapes dataset and has a resolution of $2048 \times 1024$ pixels. The original image is complemented with 15 crops where each crop removes $c_{l}=10$ rows from the top and the bottom as well as the 20 left-most and right-most columns of the previous crop.

\section{Nested Image Crops and Uncertainty Mea- sures}

Let $x \in \mathbb{R}^{N_{r} \times N_{c} \times 3}$ denote an RGB input image. For a chosen crop distance of $c_{l}$ we define a restriction operator $R_{i}$ that removes the $i \cdot c_{l}$ top and bottom rows as well as the $2 i \cdot c_{l}$ left and right most pixels from $x$, i.e.,

$$
\begin{aligned}
R_{i} x=\left\{x_{p, q, .}:\right. & i c_{l} \leq p<N_{r}-i c_{l}, \\
& \left.2 i c_{l} \leq p<N_{c}-2 i c_{l}\right\} .
\end{aligned}
$$

In order to re-scale a cropped image to a desired resolution, we define an interpolation operator $I_{i}^{j}$ which performs a bilinear interpolation for $R_{i} x \in \mathbb{R}^{\left(N_{r}-2 i c_{l}\right) \times\left(N_{c}-4 i c_{l}\right) \times 3}$ such that

$$
\begin{aligned}
I_{i}^{j} R_{i} x & \in \mathbb{R}^{\left(N_{r}-2 j c_{l}\right) \times\left(N_{c}-4 j c_{l}\right) \times 3} \\
\text { and } \quad I_{i}^{0} R_{i} x & \in \mathbb{R}^{N_{r} \times N_{c} \times 3} .
\end{aligned}
$$

A segmentation network with a softmax output layer can be seen as a statistical model that provides for each pixel $z$ of the image a probability distribution $f_{z}(y \mid x, w)$ on the $C$ class labels $y \in \mathcal{C}=\left\{y_{1}, \ldots, y_{C}\right\}$.

$$
P_{i}=\left(f_{z}\left(y \mid I_{i}^{0} R_{i} x, w\right)\right)_{z \in\left\{1, \ldots, N_{r}\right\} \times\left\{1, \ldots, N_{c}\right\}}
$$

for $i=0, \ldots, N_{\text {crop }}$. Note that, due to eq. (2), i.e., all inputs being equally shaped, the $P_{i}$ 's can be computed in batches which allows for efficient parallelization. In order to combine the probabilities $P_{i}$ to a common probability distribution we reshape them to their original size via

$$
Q_{i}=I_{0}^{i} P_{i} .
$$

We could now stack $Q_{i}, i=1, \ldots, N_{c r o p}$, in a pyramid fashion, sum them up and normalize the results such that we get a new probability distribution. However, this distribution would suffer from artifacts on the boundary of each
$Q_{i}$. To avoid this, we proceed as follows: Let $Z_{i}$ define a zero padding operator such that $Z_{i} Q_{i} \in \mathbb{R}^{N_{r} \times N_{c} \times C}$ and $Q_{i}$ is centered in $Z_{i} Q_{i}$ while all other entries are zero. In order to construct a smooth mean probability distribution, we introduce a kernel function $K_{i}$ that is zero where $Z_{i} Q_{i}$ is zero and equal to one where the next nested crop $Z_{i+1} Q_{i+1}$ is not equal to zero. In-between these two regions, $K_{i}$ interpolates linearly. We can now recursively define our set of probability distributions, that we will use for further investigation, by

$$
A_{0}=P_{0} \quad \text { and } \quad A_{i}=K_{i} Z_{i} Q_{i}+\left(1-K_{i}\right) A_{i-1}
$$

for $i=1, \ldots, N_{\text {crop }}$. Each of the probability distributions $A_{i}$ can be viewed as a smooth merge of the current crop and the combination of all previously merged crops, due to their recursive definition being merged smoothly as well.

In the following we generate uncertainty heat maps for each $A_{i}$ by defining pixel-wise dispersion measures. Let

$$
\hat{y}_{z}\left(A_{i}\right)=\underset{y \in \mathcal{C}}{\arg \max } A_{i, z, y} .
$$

denote the predicted class, for each pixel $z$ we define the entropy (also known as Shannon information [18]) $E_{z}$, the probability margin $M_{z}$ and the variation ratio $V_{z}$ by

$$
\begin{aligned}
E_{z}\left(A_{i}\right) & =-\frac{1}{\log (C)} \sum_{y \in \mathcal{C}} A_{i, z, y} \log A_{i, z, y}, \\
M_{z}\left(A_{i}\right) & =1-A_{i, z, \hat{y}_{z}\left(A_{i}\right)}+\max _{y \in \mathcal{C} \backslash\left\{\hat{y}_{z}\left(A_{i}\right)\right\}} A_{i, z, y}, \\
V_{z}\left(A_{i}\right) & =1-A_{i, z, \hat{y}_{z}\left(A_{i}\right)} .
\end{aligned}
$$

For each of these uncertainty measures $U_{z} \in\left\{E_{z}, M_{z}, V_{z}\right\}$ we define a mean and a variance over the number of crops

$$
\begin{aligned}
\mu U_{z} & =\frac{1}{N_{\text {crop }}} \sum_{i=0}^{N_{\text {crop }}} U_{z}\left(A_{i}\right) \\
\text { and } \quad v U_{z} & =\mu\left(U_{z}^{2}\right)-\mu\left(U_{z}\right)^{2}
\end{aligned}
$$

Furthermore we also consider a symmetrized version of the Kullback-Leibler divergence of the mean probabilities $A=$ $\frac{1}{N_{\text {crop }}} \sum_{i=0}^{N_{\text {crop }}} A_{i}$ and the original probabilities $A_{0}$ without incorporation of additional crops, i.e.,

$$
\begin{gathered}
K_{z}\left(A, A_{0}\right)=\frac{1}{2}\left(D_{K L}\left(A_{z} \| A_{0, z}\right)+D_{K L}\left(A_{0, z} \| A_{z}\right)\right) \\
=\frac{1}{2 C} \sum_{y \in \mathcal{C}} A_{z, y} \log \left(\frac{A_{0, z, y}}{A_{z, y}}\right)+A_{0, z, y} \log \left(\frac{A_{z, y}}{A_{0, z, y}}\right) .
\end{gathered}
$$

A visualization of $\mu M_{z}$ and $v M_{z}$ is given in fig. 3. The heat maps $E_{z}, M_{z}, V_{z}$ and $K_{z}$ are subject to segment-wise investigation. 


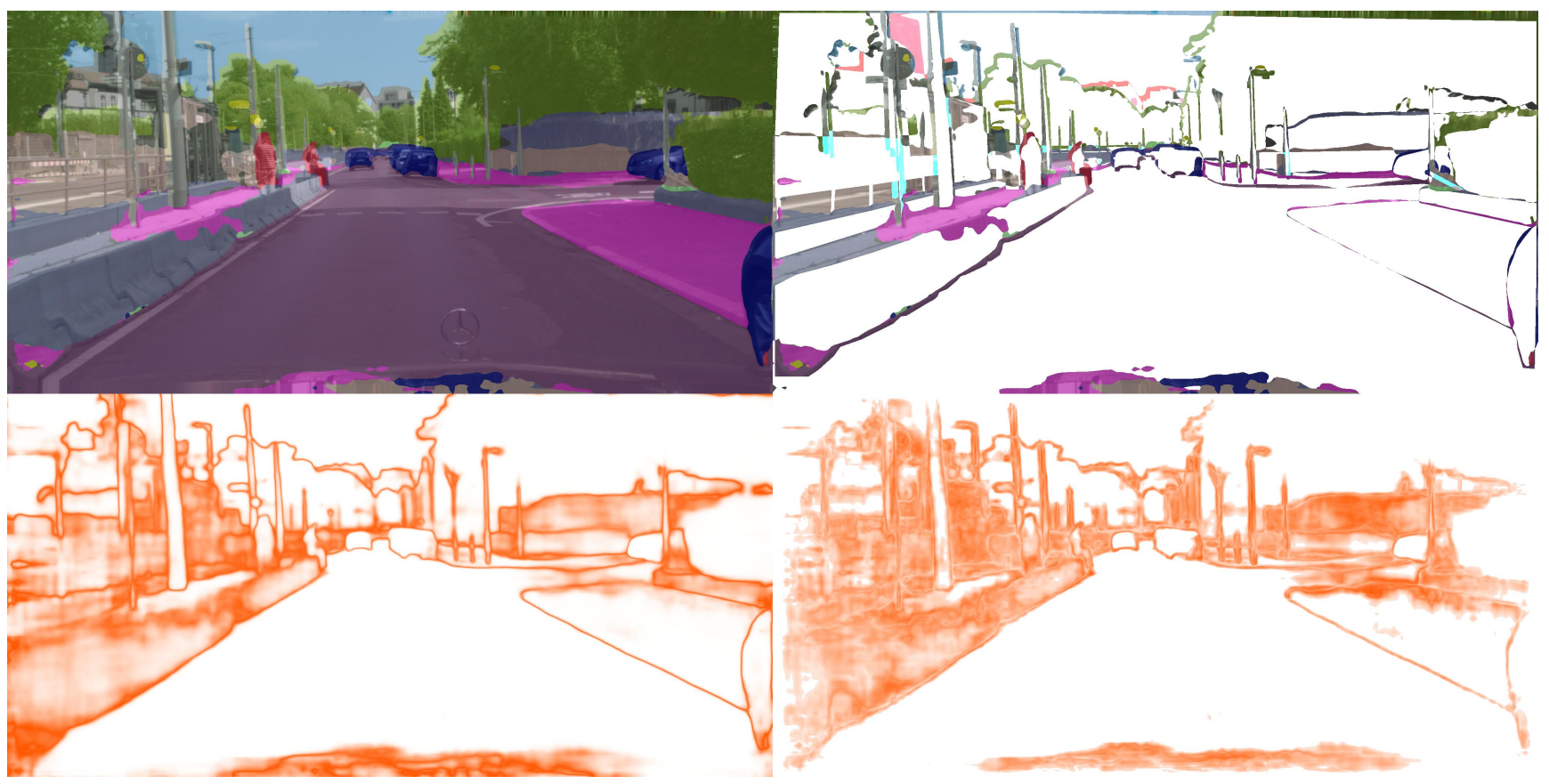

Figure 3. (Top left): segmentation $y_{z}(x)$ predicted by the neural network, (top right): predicted segmentation $y_{z}(x)$ where prediction and ground truth differ, note that the ego car is excluded from the ground truth, (bottom left): mean $\mu M_{z}$ of all probability margin heat maps, (bottom right): variance $v M_{z}$ of probability margin heat maps.

\section{Metrics Aggregated over Segments}

For a given image $x$ we define the set of connected components (segments) in the predicted segmentation $\hat{\mathcal{S}}_{x}=$ $\left\{\hat{y}_{z}(A) \mid z \in x\right\}$ by $\hat{\mathcal{K}}_{x}$. Analogously we denote by $\mathcal{K}_{x}$ the set of connected components in the ground truth $\mathcal{S}_{x}$. For each $k \in \hat{\mathcal{K}}_{x}$, we define the following quantities:

- the interior $k_{i n} \subset k$ where a pixel $z$ is an element of $k_{i n}$ if all eight neighbouring pixels are an element of $k$

- the boundary $k_{b d}=k \backslash k_{i n}$

- the intersection over union $I o U$ : let $\left.\mathcal{K}_{x}\right|_{k}$ be the set of all $k^{\prime} \in \mathcal{K}_{x}$ that have non-trivial intersection with $k$ and whose class label equals the predicted class for $k$, then

$$
\operatorname{IoU}(k)=\frac{\left|k \cap K^{\prime}\right|}{\left|k \cup K^{\prime}\right|}, \quad K^{\prime}=\bigcup_{\left.k^{\prime} \in \mathcal{K}_{x}\right|_{k}} k^{\prime}
$$

- adjusted $I o U_{\text {adj }}$ : let $Q=\left\{q \in \hat{\mathcal{K}}_{x}: q \cap K^{\prime} \neq \emptyset\right\}$, as in [16] we use in our tests

$$
I_{o} U_{\text {adj }}(k)=\frac{\left|k \cap K^{\prime}\right|}{\left|k \cup\left(K^{\prime} \backslash Q\right)\right|}
$$

- the pixel sizes $S=|k|, S_{i n}=\left|k_{i n}\right|, S_{b d}=\left|k_{b d}\right|$

- the mean dispersion $\bar{D}, \bar{D}_{i n}, \bar{D}_{b d}$ defined as

$$
\bar{D}_{\sharp}(k)=\frac{1}{S_{\sharp}} \sum_{z \in k_{\sharp}} D_{z}(x), \quad \sharp \in\{, \text { in }, b d\}
$$

where $D_{z} \in\left\{K_{z}, \mu U_{z}, v U_{z}: U_{z}=E_{z}, M_{z}, V_{z}\right\}$

- the relative sizes $\tilde{S}=S / S_{b d}, \tilde{S}_{i n}=S_{i n} / S_{b d}$

- the relative mean dispersions $\tilde{\bar{D}}=\bar{D} \tilde{S}, \tilde{\bar{D}}_{i n}=$ $\bar{D}_{i n} \tilde{S}_{i n}$

- the geometric center $\bar{k}=\left(\bar{k}_{1}, \bar{k}_{2}\right)=\frac{1}{S} \sum_{z \in k}\left(z_{1}, z_{2}\right)$ where $z_{1}$ and $z_{2}$ are the vertical and horizontal coordinates of the pixel $z$ in $x$, respectively

- the mean class probabilities for each class $y \in$ $\{1, \ldots, C\}$

$$
P_{y}(k)=\frac{1}{S} \sum_{z \in k} A_{z, y}
$$

- sets of metrics

$$
\tau U=\left\{\tau \bar{U}, \tau \bar{U}_{b d}, \tau \bar{U}_{i n}, \tau \tilde{\bar{U}}, \tau \tilde{\bar{U}}_{i n}\right\}
$$

for $\tau \in\{\mu, v\}$ and $U \in\{V, M, E\}$ as well as

$$
P=\left\{P_{y}: y=1, \ldots, C\right\}, \Sigma=\left\{S, S_{i n}, S_{b d}, \tilde{S}, \tilde{S}_{i n}\right\}
$$

Typically, $D_{z}$ is large for $z \in k_{b d}$. This motivates the separate treatment of interior and boundary in all dispersion measures. Furthermore we observe that bad or wrong predictions often come with fractal segment shapes (which have a relatively large amount of boundary pixels, measurable by $\tilde{S}=S / S_{b d}$ and $\left.\tilde{S}_{i n}=S_{i n} / S_{b d}\right)$ and/or high dispersions $\bar{D}_{i n}$ on the segment's interior. With the exception 


\begin{tabular}{|c|c|c|c|c|c|c|c|c|c|c|}
\hline & \multicolumn{5}{|c|}{ all $2048 \times 1024$ pixels } & \multicolumn{5}{|c|}{$1024 \times 512$ center section } \\
\hline number of crops & 1 & 2 & 4 & 8 & 16 & 1 & 2 & 4 & 8 & 16 \\
\hline $0:$ road & $95.94 \%$ & $96.00 \%$ & $96.04 \%$ & $96.10 \%$ & $96.23 \%$ & $95.00 \%$ & $95.05 \%$ & $95.13 \%$ & $95.25 \%$ & $95.52 \%$ \\
\hline 1: sidewalk & $71.83 \%$ & $72.08 \%$ & $72.31 \%$ & $72.63 \%$ & $73.26 \%$ & $62.58 \%$ & $62.88 \%$ & $63.27 \%$ & $63.91 \%$ & $65.30 \%$ \\
\hline 2: building & $84.83 \%$ & $85.01 \%$ & $85.15 \%$ & $85.32 \%$ & $85.58 \%$ & $76.79 \%$ & $77.07 \%$ & $77.33 \%$ & $77.70 \%$ & $78.43 \%$ \\
\hline 3: wall & $34.41 \%$ & $34.48 \%$ & $34.40 \%$ & $34.22 \%$ & $33.92 \%$ & $32.55 \%$ & $32.97 \%$ & $32.98 \%$ & $33.12 \%$ & $32.97 \%$ \\
\hline 4: fence & $49.23 \%$ & $49.92 \%$ & $49.96 \%$ & $50.33 \%$ & $50.49 \%$ & $41.07 \%$ & $41.48 \%$ & $41.47 \%$ & $42.24 \%$ & $42.90 \%$ \\
\hline 5: pole & $28.97 \%$ & $29.45 \%$ & $29.89 \%$ & $30.55 \%$ & $31.70 \%$ & $22.06 \%$ & $22.50 \%$ & $22.90 \%$ & $23.72 \%$ & $25.59 \%$ \\
\hline 6: traffic light & $41.70 \%$ & $42.35 \%$ & $42.72 \%$ & $43.28 \%$ & $44.23 \%$ & $26.40 \%$ & $27.56 \%$ & $28.10 \%$ & $29.00 \%$ & $30.85 \%$ \\
\hline 7: traffic sign & $50.59 \%$ & $50.94 \%$ & $51.45 \%$ & $52.08 \%$ & $53.27 \%$ & $39.54 \%$ & $40.08 \%$ & $40.95 \%$ & $41.88 \%$ & $44.03 \%$ \\
\hline 8: vegetation & $84.43 \%$ & $84.58 \%$ & $84.72 \%$ & $84.90 \%$ & $85.23 \%$ & $77.39 \%$ & $77.65 \%$ & $77.92 \%$ & $78.31 \%$ & $79.07 \%$ \\
\hline 9: terrain & $52.88 \%$ & $53.25 \%$ & $53.43 \%$ & $53.44 \%$ & $53.69 \%$ & $43.88 \%$ & $44.46 \%$ & $45.08 \%$ & $45.49 \%$ & $46.25 \%$ \\
\hline 10: sky & $82.82 \%$ & $82.91 \%$ & $82.98 \%$ & $83.16 \%$ & $83.40 \%$ & $64.91 \%$ & $65.07 \%$ & $65.25 \%$ & $65.83 \%$ & $67.20 \%$ \\
\hline 11: person & $63.40 \%$ & $63.85 \%$ & $64.21 \%$ & $64.93 \%$ & $66.11 \%$ & $63.25 \%$ & $63.74 \%$ & $64.20 \%$ & $65.06 \%$ & $66.69 \%$ \\
\hline 12: rider & $43.63 \%$ & $43.90 \%$ & $44.08 \%$ & $44.50 \%$ & $45.41 \%$ & $42.53 \%$ & $42.85 \%$ & $43.15 \%$ & $44.01 \%$ & $45.41 \%$ \\
\hline 13: car & $85.06 \%$ & $85.20 \%$ & $85.40 \%$ & $85.69 \%$ & $86.23 \%$ & $79.38 \%$ & $79.58 \%$ & $79.87 \%$ & $80.37 \%$ & $81.37 \%$ \\
\hline 14: truck & $66.64 \%$ & $66.49 \%$ & $66.41 \%$ & $65.82 \%$ & $64.16 \%$ & $66.97 \%$ & $67.54 \%$ & $67.44 \%$ & $67.56 \%$ & $67.00 \%$ \\
\hline 15: bus & $70.47 \%$ & $70.56 \%$ & $70.56 \%$ & $70.38 \%$ & $70.22 \%$ & $70.95 \%$ & $71.17 \%$ & $71.46 \%$ & $71.60 \%$ & $71.85 \%$ \\
\hline 16: train & $58.44 \%$ & $59.63 \%$ & $59.92 \%$ & $58.87 \%$ & $57.63 \%$ & $58.44 \%$ & $59.46 \%$ & $60.51 \%$ & $60.00 \%$ & $61.15 \%$ \\
\hline 17: motorcycle & $48.16 \%$ & $48.37 \%$ & $48.63 \%$ & $49.32 \%$ & $50.21 \%$ & $45.21 \%$ & $45.49 \%$ & $46.43 \%$ & $47.28 \%$ & $48.57 \%$ \\
\hline 18: bicycle & $61.74 \%$ & $62.09 \%$ & $62.44 \%$ & $63.01 \%$ & $63.94 \%$ & $55.22 \%$ & $55.73 \%$ & $56.28 \%$ & $57.09 \%$ & $58.65 \%$ \\
\hline$m I o U$ & $61.85 \%$ & $62.16 \%$ & $62.35 \%$ & $62.55 \%$ & $62.89 \%$ & $56.01 \%$ & $56.44 \%$ & $56.83 \%$ & $57.34 \%$ & $58.36 \%$ \\
\hline
\end{tabular}

Table 1. The (classical) $I o U$ for each class over the whole dataset as well as the mean $I o U$ ( $m I o U$ ) over all classes, both as a function of the number of crops. These numbers are computed once for the entire images of $2048 \times 1024$ pixels (left half) and once for the center section containing $1024 \times 512$ pixels (right-hand half). The best results for each class are highlighted.

\begin{tabular}{|l|c||l|r||l|c||l|r|}
\hline$\mu \bar{E}$ & $\mathbf{- 0 . 7 1 3 4 0}$ & $v \bar{E}^{*}$ & -0.18668 & $\mu \bar{M}$ & $\mathbf{- 0 . 8 4 3 5 8}$ & $v \bar{M}^{*}$ & -0.30971 \\
$\mu \bar{E}_{b d}$ & -0.43822 & $v \bar{E}_{b d}^{*}$ & -0.14376 & $\mu \bar{M}_{b d}$ & -0.48518 & $v \bar{M}_{b d}^{*}$ & +0.08374 \\
$\mu \bar{E}_{i n}$ & $\mathbf{- 0 . 7 1 4 2 2}$ & $v \bar{E}_{i n}^{*}$ & -0.19332 & $\mu \bar{M}_{i n}$ & $\mathbf{- 0 . 8 3 1 8 3}$ & $v \bar{M}_{i n}^{*}$ & -0.32423 \\
$\mu \tilde{\bar{E}}^{*}$ & +0.34611 & $v \tilde{\bar{E}}^{*}$ & +0.33995 & $\mu \tilde{\bar{M}}^{2}$ & +0.30129 & $v \tilde{\bar{M}}^{*}$ & +0.34914 \\
$\mu \tilde{\bar{E}}_{i n}$ & +0.40510 & $v \tilde{\bar{E}}_{i n}^{*}$ & +0.37059 & $\mu \tilde{\bar{M}}_{i n}$ & +0.34028 & $v \tilde{\bar{M}}_{i n}^{*}$ & +0.35836 \\
\hline$\mu \bar{V}^{*}$ & $\mathbf{- 0 . 7 9 5 4 6}$ & $v \bar{V}^{*}$ & -0.36141 & $\bar{K}^{*}$ & -0.33353 & $S$ & +0.45958 \\
$\mu \bar{V}_{b d}^{*}$ & $\mathbf{- 0 . 5 0 2 1 8}$ & $v \bar{V}_{b d}^{*}$ & -0.05362 & $\bar{K}_{b d}^{*}$ & -0.12983 & $S_{b d}$ & $\mathbf{+ 0 . 6 0 3 6 7}$ \\
$\mu \bar{V}_{i n}^{*}$ & $\mathbf{- 0 . 7 8 5 7 8}$ & $v \bar{V}_{i n}^{*}$ & -0.36814 & $\bar{K}_{i n}^{*}$ & -0.32906 & $S_{i n}$ & +0.45705 \\
$\mu \tilde{\bar{V}}^{*}$ & +0.25307 & $v \tilde{\bar{V}}^{*}$ & +0.29991 & $\tilde{\bar{K}}^{*}$ & +0.17631 & $\tilde{S}$ & $\mathbf{+ 0 . 6 8 6 3 6}$ \\
$\mu \tilde{V}_{i n}^{*}$ & +0.31223 & $v \tilde{V}_{i n}^{*}$ & +0.32238 & $\tilde{\bar{K}}_{i n}^{*}$ & +0.21686 & $\tilde{S}_{i n}$ & $\mathbf{+ 0 . 6 8 6 3 6}$ \\
\hline $\bar{k}_{1}^{*}$ & -0.05955 & $\bar{k}_{2}^{*}$ & +0.14190 & & & & \\
\hline
\end{tabular}

Table 2. Pearson correlation coefficients for all constructed segment-wise metrics. All metrics marked with $\mathrm{a}^{*}$ were not used in [16]. All results with bsolute values greater than 0.5 are highlighted.

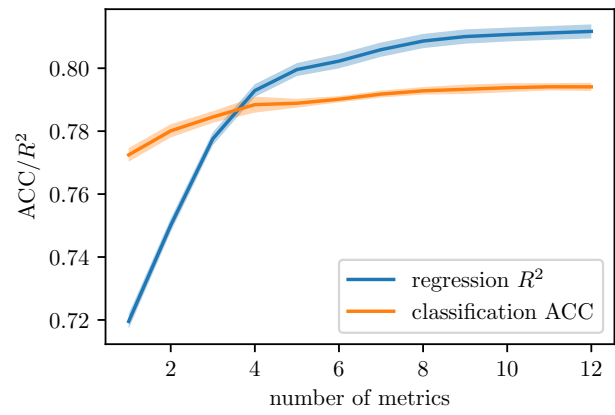

Figure 4. Meta classification accuracy and meta regression $R^{2}$, both as a function of the number of metrics (sets stated in table 4). Results averaged over 10 runs, the shaded regions depict the corresponding standard deviation. of $I o U$ and $I o U_{\text {adj }}$, all scalar quantities defined above can be computed without the knowledge of the ground truth. Our aim is to analyze to which extent they are suited for the tasks of meta classification and meta regression for the $I o U_{\text {adj }}$.

\section{Numerical Experiments: Street Scenes}

In this section we investigate the properties of the nested crops and the metrics defined in the previous sections for the example of a semantic segmentation of street scenes. To this end, we consider the DeepLabv3+ network [3] with MobilenetV2 [17] encoder for which we use a reference im- plementation in Tensorflow [1] as well as weights pretrained on the Cityscapes dataset [4] (available on GitHub). As parameters for the DeepLabv3+ framework we use an output stride of 16 , the input image is evaluated within the framework only on its original scale. These parameters result in a mean $I o U$ of $61.85 \%$ on the Cityscapes validation set, here mean refers to mean over all classes. We refer to [3] for a detailed explanation of the chosen parameters.

For our tests we produced $N_{\text {crop }}=15$ crops, i.e., we have 16 nested images for each original image. The Cityscapes validation dataset contains 500 images with a resolution of $2048 \times 1024$ pixels. Each crop is obtained from the previous one by removing the 20 left-most and 


\begin{tabular}{|c|c|c|c|c|c|c|}
\hline \multicolumn{7}{|c|}{ Meta Classification $I o U_{a d j}=0,>0$} \\
\hline & \multicolumn{2}{|c|}{ entropy } & \multicolumn{2}{|c|}{ probability margin } & \multirow{2}{*}{$\begin{array}{c}\text { class probabilities } \\
P\end{array}$} & \\
\hline & $\mu E \cup v E$ & $\mu E$ & $\mu M \cup v M$ & $\mu M$ & & \\
\hline ACC & $77.82 \%( \pm 0.26 \%)$ & $77.06 \%( \pm 0.26 \%)$ & $78.49 \%( \pm 0.28 \%)$ & $76.99 \%( \pm 0.27 \%)$ & $64.70 \%( \pm 0.36 \%)$ & \\
\hline AUROC & $85.39 \%( \pm 0.21 \%)$ & $84.66 \%( \pm 0.19 \%)$ & $85.47 \%( \pm 0.21 \%)$ & $85.06 \%( \pm 0.22 \%)$ & $64.65 \%( \pm 0.34 \%)$ & \\
\hline \multicolumn{7}{|c|}{ Meta Regression $I o U_{a d j}$} \\
\hline$\sigma$ & $0.162( \pm 0.001)$ & $0.163( \pm 0.001)$ & $0.147( \pm 0.001)$ & $0.150( \pm 0.001)$ & $0.276( \pm 0.001)$ & \\
\hline$R^{2}$ & $73.59 \%( \pm 0.29 \%)$ & $73.10 \%( \pm 0.28 \%)$ & $78.27 \%( \pm 0.24 \%)$ & $77.34 \%( \pm 0.23 \%)$ & $22.92 \%( \pm 0.32 \%)$ & \\
\hline \multicolumn{7}{|c|}{ Meta Classification $I o U_{a d j}=0,>0$} \\
\hline & \multicolumn{2}{|c|}{ variation ratio } & \multicolumn{2}{|c|}{ segment sizes } & \multicolumn{2}{|c|}{ all metrics } \\
\hline & $\mu V \cup v V$ & $\mu V$ & $\Sigma \cup\left\{\bar{k}_{1}, \bar{k}_{2}\right\}$ & $\Sigma$ & with variances & without \\
\hline ACC & $78.14 \%( \pm 0.25 \%)$ & $76.96 \%( \pm 0.24 \%)$ & $77.60 \%( \pm 0.17 \%)$ & $77.25 \%( \pm 0.23 \%)$ & $79.58 \%( \pm 0.15 \%)$ & $79.30 \%( \pm 0.11 \%)$ \\
\hline AUROC & $85.41 \%( \pm 0.21 \%)$ & $84.89 \%( \pm 0.21 \%)$ & $84.94 \%( \pm 0.17 \%)$ & $84.36 \%( \pm 0.25 \%)$ & $87.38 \%( \pm 0.16 \%)$ & $87.08 \%( \pm 0.16 \%)$ \\
\hline \multicolumn{7}{|c|}{ Meta Regression $I o U_{a d j}$} \\
\hline$\sigma$ & $0.154( \pm 0.001)$ & $0.156( \pm 0.001)$ & $0.174( \pm 0.001)$ & $0.179( \pm 0.001)$ & $0.135( \pm 0.001)$ & $0.136( \pm 0.001)$ \\
\hline$R^{2}$ & $76.12 \%( \pm 0.26 \%)$ & $75.50 \%( \pm 0.26 \%)$ & $69.41 \%( \pm 0.27 \%)$ & $67.79 \%( \pm 0.27 \%)$ & $81.71 \%( \pm 0.20 \%)$ & $81.36 \%( \pm 0.19 \%)$ \\
\hline
\end{tabular}

Table 3. Comparison of sets of metrics. Each of the uncertainty heat map based set of metrics is used once including the variance metrics $(\mu U \cup v U$ for $U=E, M, V)$ and once without variance based metrics $(\mu U$ for $U=E, M, V)$. We state results for the segments sizes $\Sigma$ including the geometric center $\bar{k}$ and without. The average predicted class probabilities $P$ are given by 19 metrics, one for each class. All results are calculated on the metrics' validation set, the best results are highlighted.

\begin{tabular}{||l||c|c|c|c|c|c|c|c|c|c|c|c|c||}
\hline number of metrics & 1 & 2 & 3 & 4 & 5 & 6 & 7 & 8 & 9 & 10 & 11 & 12 & 61 \\
\hline classification accuracy (in \%) & 0.7725 & 0.7801 & 0.7845 & 0.7884 & 0.7889 & 0.7901 & 0.7918 & 0.7928 & 0.7933 & 0.7938 & 0.7941 & 0.7944 & 0.7958 \\
added metric & $\tilde{S}$ & $v \hat{M}_{i n}$ & $\bar{k}_{2}$ & $P_{4}$ & $P_{5}$ & $v \tilde{\hat{E}}_{i n}$ & $P_{14}$ & $P_{17}$ & $P_{15}$ & $P_{3}$ & $\mu \bar{M}_{b d}$ & $v \tilde{\hat{E}}^{2}$ & all \\
\hline regression $R^{2}$ (in \%) & 0.7195 & 0.7501 & 0.7776 & 0.7929 & 0.8000 & 0.8023 & 0.8059 & 0.8086 & 0.8101 & 0.8107 & 0.8112 & 0.8117 & 0.8171 \\
added metric & $\mu \bar{M}$ & $\tilde{S}$ & $\mu \bar{M}_{b d}$ & $\bar{k}_{2}$ & $\mu \bar{M}_{i n}$ & $v \hat{M}_{b d}$ & $v \tilde{\hat{E}}$ & $P_{5}$ & $P_{11}$ & $P_{18}$ & $P_{0}$ & $\tilde{\bar{K}}_{\text {in }}$ & all \\
\hline
\end{tabular}

Table 4. Metric selection using a greedy method that adds in each step one metric that maximizes the meta classification/regression performance. The upper part of the table contains the sequence of metrics added corresponding to classification accuracy maximization, the lower one corresponding to $R^{2}$ maximization. All results are calculated on the metrics' validation set.

the 20 right-most columns as well as the 10 top and the 10 bottom rows. In all tests we only consider segments with non-empty interior. For the combined prediction using all 16 crops, MobilenetV2 predicts 46896 segments of which 38811 have non-empty interior. From those segments with non-empty interior, 24354 have $I o U_{\text {adj }}>0$. This gives a meta classification accuracy baseline of $62.75 \%$ if we predict that each segment has $I_{o} U_{\text {adj }}>0$. Note that, when only using the prediction of the original image, we obtain 53424 components, 42261 with non-empty interior of which 24590 have $I o U_{\text {adj }}>0$ (resulting in $58.19 \%$ meta classification baseline accuracy). Thus, meta classification results for different numbers of crops are not straight forward comparable. Hence, we focus on results for 16 crops in the following studies.

All results, if not stated otherwise, were computed from 10 repeated runs where training and validation sets (both of the same size) were re-sampled. We give mean results as well as corresponding standard deviations in brackets.

Performance depending on the number of crops. Table 1 contains the values for the classical $I o U$ over the whole Cityscapes validation dataset for the different classes as a function of the number of crops $(1,2,4,8,16)$, for the en- tire image $(2048 \times 1024$ pixels $)$ as well as for the $1024 \times 512$ center pixels. In both cases the mIoU increases continuously when adding further crops. For the whole image the $m I o U$ increases from $61.85 \%$ to $62.89 \%$ (i.e., by 1.04 percentage points (pp)) and for the center section from $56.01 \%$ to $58.36 \%$ (by $2.35 \mathrm{pp}$ ). This demonstrates that our crop based method indeed has the desired effect on smaller objects further away from ego car. For classes of particular interest, like person, rider and traffic sign, we observe improvements in the center section of 2.88 (for rider) to $4.49 \mathrm{pp}$ (for traffic sign). We make these observations even though the original image is presented to the segmentation network at full resolution and the zoomed crops do not contain any additional information. In summary these results already justify the deployment of our approach which can be nicely parallelized over the data batch. In addition we obtain further uncertainty information which we investigate in the subsequent paragraphs.

Correlation of segment-wise metrics with the $I o U_{\text {adj }}$. Table 2 contains the Pearson correlation coefficients for all segment-wise metrics for all 16 available image crops constructed in section 3 . We observe strong correlations for the measures $\bar{D}$ and $\bar{D}_{i n}$ where $D \in\{\mu M, \mu V, \mu E\}$ and for the 


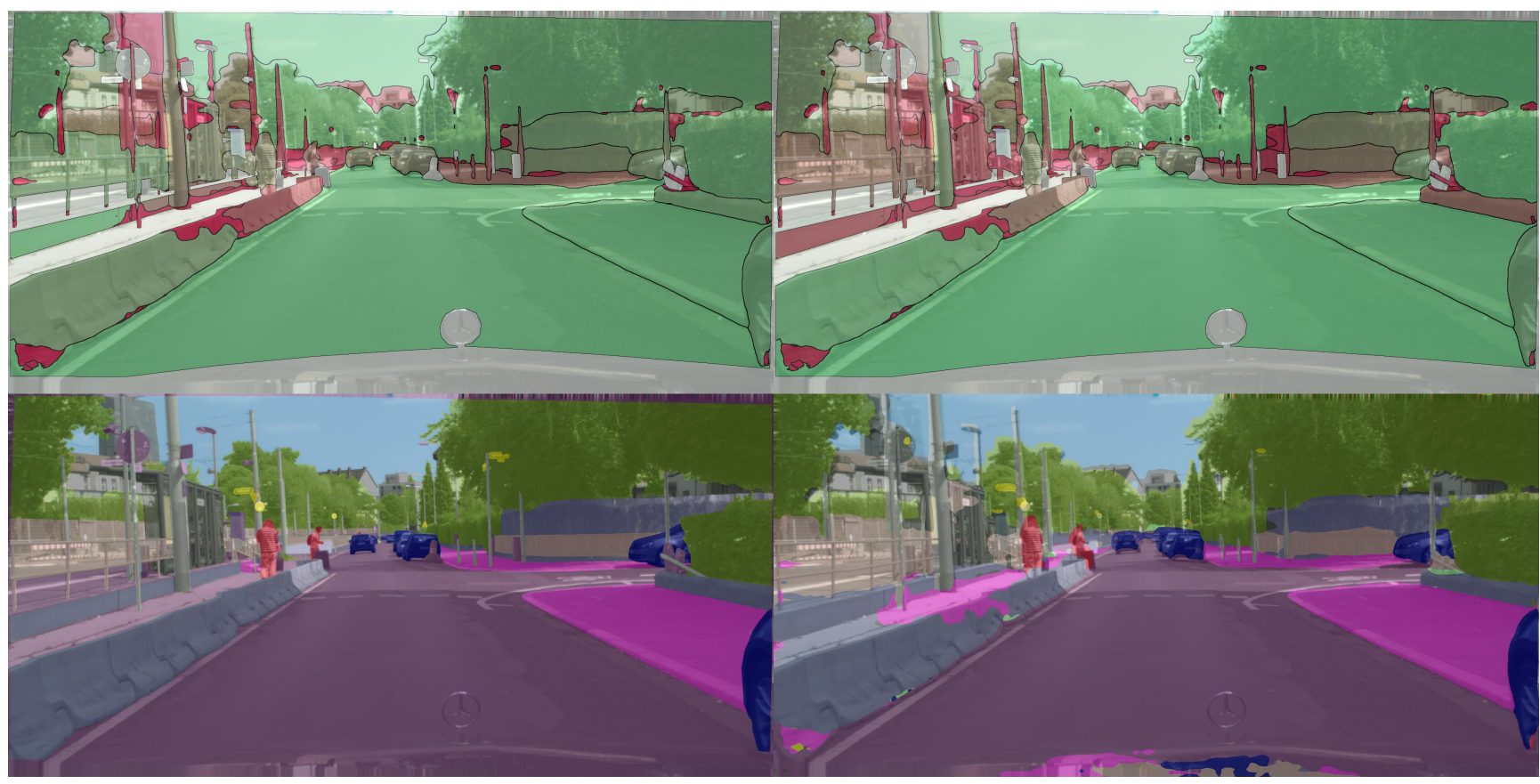

Figure 5. Prediction of the $I o U_{\text {adj }}$ via linear regression. (bottom left): ground truth, (bottom right): predicted segments, (top left): true $I o U_{\mathrm{adj}}$ for the predicted segments and (top right): predicted $I o U_{\mathrm{adj}}$ for the predicted segments. In the top row, green color corresponds to high $I o U_{\text {adj }}$ values and red color to low ones, for the white regions there is no ground truth available. These regions are excluded from the statistical evaluation.

\begin{tabular}{|c|c|c|c|c|c|c|}
\hline \multicolumn{7}{|c|}{ Meta Classification $I o U_{a d j}=0,>0$} \\
\hline & \multicolumn{2}{|c|}{ all metrics } & \multicolumn{2}{|c|}{ metrics from [16] } & \multicolumn{2}{|c|}{ entropy baseline } \\
\hline & train & val & train & val & train & val \\
\hline ACC & $79.88 \%( \pm 0.21 \%)$ & $79.58 \%( \pm 0.15 \%)$ & $77.37 \%( \pm 0.28 \%)$ & $77.24 \%( \pm 0.29 \%)$ & $70.16 \%( \pm 0.22 \%)$ & $70.16 \%( \pm 0.22 \%)$ \\
\hline AUROC & $87.61 \%( \pm 0.16 \%)$ & $87.38 \%( \pm 0.16 \%)$ & $85.32 \%( \pm 0.22 \%)$ & $85.18 \%( \pm 0.20 \%)$ & $77.73 \%( \pm 0.21 \%)$ & $77.69 \%( \pm 0.21 \%)$ \\
\hline \multicolumn{7}{|c|}{ Meta Regression $I o U_{a d j}$} \\
\hline$\sigma$ & $0.135( \pm 0.001)$ & $0.135( \pm 0.001)$ & $0.144( \pm 0.001)$ & $0.144( \pm 0.001)$ & $0.213( \pm 0.001)$ & $0.213( \pm 0.001)$ \\
\hline$R^{2}$ & $81.72 \%( \pm 0.22 \%)$ & $81.71 \%( \pm 0.20 \%)$ & $79.00 \%( \pm 0.20 \%)$ & $79.08 \%( \pm 0.20 \%)$ & $54.30 \%( \pm 0.40 \%)$ & $54.43 \%( \pm 0.28 \%)$ \\
\hline
\end{tabular}

Table 5. Results for all for meta classification and regression for three different sets of metrics. (Left block): all metrics including variance metrics, (center block): all metrics but without variances, (right-hand block): set of metrics used in [16], i.e., not marked with a * in table 2. The best results for the validation set are highlighted.

relative size measures $\tilde{S}$ and $\tilde{S}_{i n}$. All other size measures as well as $\mu D_{b d}$ for $D \in\{M, V, E\}$ also show increased correlation coefficients. The variances and the KullbackLeibler measures seem to play a minor role, however they might contribute additional information for a model that predicts the $I o U_{\text {adj }}$.

\section{Metric selection for meta classification and meta regres-} sion. In table 3 we compare different subsets of metrics. For the tasks of meta classification, we do so in terms of meta classification accuracy ( $I o U_{\text {adj }}=0$ vs. $I o U_{\text {adj }}>0$ ) and in terms of the area under curve corresponding to the receiver operator characteristic curve (AUROC, see [5]). The receiver operator characteristic curve is obtained by varying the decision threshold of the classification output for deciding whether $I o U_{\text {adj }}=0$ or $I o U_{\text {adj }}>0$. For the task of meta regression we state resulting standard deviations $\sigma$ of the linear regression fit's residual as well as $R^{2}$ values. We observe that the probability margin heat map yields the most predictive set of metrics, closely followed by the variation ratio. Altogether all heat maps yield fairly similar results and also the segment sizes yield a strong predictive set. The mean class probabilities $P$ by itself are not predictive enough, at least for linear and logistic regression models as being used here. In all cases we observe a significant performance increase when incorporating the variance based heat maps, also the geometric center yields valuable extra information. When using all metrics together, another significant increase in all performance measures can be observed. Noteworthily, we obtain AUROC values of up to $87.38 \%$ for 
meta classification and $R^{2}$ values of up to $81.71 \%$ for meta regression which demonstrates the predictive power of our metrics. When omitting the variance based metrics, the performance can not be maintained entirely, i.e., we observe a slight decrease of 0.28 to $0.35 \mathrm{pp}$ in all accuracy measures. A visual demonstration of the meta regression performance can be found in fig. 5 .

In order to further analyze the different subsets of metrics, we perform a greedy heuristic. We start with an empty set of metrics and add iteratively a single metric that improves meta prediction performance maximally. We perform this test twice, once for meta classification accuracy and once for meta regression $R^{2}$. Figure 4 depicts both performance measures as functions of the number of metrics. In both cases the curves stagnate quite quickly, indicating that a small set of metrics might be sufficient for a good model. This is confirmed by the results stated in table 4 . For the meta regression four of the first six metrics are variants of the probability margin. Combined with the geometric center $k_{2}$ and the relative segment size $\tilde{S}$, this set obtains an $R^{2}$ of $80.23 \%$. Adding the rest of the metrics to this set only results in an increase of 1.48 pp to the final $R^{2}$ of $81.71 \%$. For the meta classification we start with $\tilde{S}$ at $77.25 \%$ classification accuracy which is only 2.33 pp below the accuracy for all metrics. Six out of the first ten added metrics are class probabilities and already after the seventh metric we obtain a classification accuracy of $79.18 \%$. In both cases, for meta classification and regression, a small subset of metrics can be determined such that the corresponding performance is close to the performance for the full set of metrics. Also in both cases the variation ratio heat map $V_{z}$ is not required.

Comparison with baseline approaches and others. In table 5 we compare our results for all metrics with the set metrics introduced in [16] (cf. table 2) and an entropy baseline where only a single entropy metric $\mu \bar{E}$ is employed. We do so as the entropy is a very commonly used uncertainty measure. In terms of AUROC we obtain an improvement of $2.20 \mathrm{pp}$ and in terms of $R^{2}$ of $2.63 \mathrm{pp}$. When comparing the full set of metrics with the entropy baseline we obtain very pronounced gaps, $9.69 \mathrm{pp}$ in AUROC and $27.28 \mathrm{pp}$ in $R^{2}$. In all three cases training and validation accuracies are tight, i.e., we do not observe any overfitting issues.

Meta classification and regression with neural networks. We repeat the tests from table 5 for all metrics, however this time we use neural networks for meta classification and regression. Our neural networks are equipped with two hidden layers containing 61 neurons each and we employ $\ell_{2}$ regularization with $\lambda=0.005$, results are stated in table 6 . The difference between training and validation accuracies indicates that the neural network is slightly overfitting. When deploying neural networks instead of linear

\begin{tabular}{||l||c|c||c||}
\hline \multicolumn{4}{||c||}{ Classification $I o U_{a d j}=0,>0$} \\
\hline \multicolumn{1}{||c||}{} & neural networks & linear models \\
\hline \multicolumn{1}{||c||}{} & train & val & val \\
ACC & $83.22 \%( \pm 0.15 \%)$ & $\mathbf{8 1 . 9 3 \%}( \pm \mathbf{0 . 2 2} \%)$ & $79.58 \%( \pm 0.15 \%)$ \\
AUROC & $91.00 \%( \pm 0.11 \%)$ & $\mathbf{8 9 . 8 9 \%}( \pm \mathbf{0 . 0 7 \%})$ & $87.38 \%( \pm 0.16 \%)$ \\
\hline \multicolumn{4}{c|}{ Regression $I o U_{a d j}$} \\
\hline$\sigma$ & $0.120( \pm 0.000)$ & $\mathbf{0 . 1 2 3}( \pm \mathbf{0 . 0 0 1})$ & $0.135( \pm 0.001)$ \\
$R^{2}$ & $85.48 \%( \pm 0.07 \%)$ & $\mathbf{8 4 . 7 7 \%}( \pm \mathbf{0 . 3 0 \%})$ & $81.71 \%( \pm 0.20 \%)$ \\
\hline
\end{tabular}

Table 6. Results obtained from a neural network used for meta classification and meta regression with all metrics. For simpler comparison we state the validation accuracies for linear models. The best results for the validation set are highlighted.

models, the validation accuracy increases by $2.35 \mathrm{pp}$ and the validation AUROC by $2.51 \mathrm{pp}$. For the meta regression, the standard deviation $\sigma$ is reduced by 0.012 and the $R^{2}$ value is increased significantly by $3.06 \mathrm{pp}$. Note that, the results for $\sigma$ may lack interpretability when using a neural network, just as the whole model trades transparency for performance.

\section{Conclusion and Outlook}

In this paper we extend the approach presented in [16]. Firstly, we introduce an approach that generates a batch of nested image crops that are presented to the segmentation network and yield a batch of probability distributions. The aggregated probabilities show improved $m I o U$ values, especially with respect to the far range section in the center of the input image. Secondly, we add segment-wise metrics constructed from variation ratio, Kullback-Leibler divergence, geometric center and crop variance based metrics. Thirdly, for the meta classification and meta regression, we replace the linear model with neural networks. All three aspects contribute to a significant improvement over the approach presented in [16]. More precisely, we obtain an increase in meta classification accuracy of $4.69 \mathrm{pp}$ and an increase of AUROC of $4.80 \mathrm{pp}$. The $R^{2}$ for meta regression is increased by $5.69 \mathrm{pp}$. Currently we are working on time-dynamic meta classification and regression approaches which make predictions from time series of metrics. As we only presented an approach for false positive detection we also plan to combine this with approaches for false negative detection, see e.g. [2]. Combining these approaches might eventually result in improved segmentation performance, at least with respect to certain classes. The source code of our method is publicly available at https: / / github. com/ mrottmann/MetaSeg/tree/nested_metaseg.

Acknowledgements. We thank Hanno Gottschalk, Peter Schlicht and Fabian Hüger for discussion and useful advice. 


\section{References}

[1] M. Abadi, A. Agarwal, P. Barham, et al. TensorFlow: Largescale machine learning on heterogeneous systems, 2015. Software available from tensorflow.org. 2, 5

[2] R. Chan, M. Rottmann, F. Hüger, P. Schlicht, and H. Gottschalk. Application of decision rules for handling class imbalance in semantic segmentation. CoRR, abs/1901.08394, 2019. 8

[3] L.-C. Chen, Y. Zhu, G. Papandreou, F. Schroff, and H. Adam. Encoder-decoder with atrous separable convolution for semantic image segmentation. CoRR, abs/1802.02611, 2018. 2,5

[4] M. Cordts, M. Omran, S. Ramos, T. Rehfeld, M. Enzweiler, R. Benenson, U. Franke, S. Roth, and B. Schiele. The cityscapes dataset for semantic urban scene understanding. In Proc. of the IEEE Conference on Computer Vision and Pattern Recognition (CVPR), 2016. 2, 5

[5] J. Davis and M. Goadrich. The relationship between precision-recall and ROC curves. In Machine Learning, Proceedings of the Twenty-Third International Conference (ICML 2006), Pittsburgh, Pennsylvania, USA, June 25-29, 2006, pages 233-240, 2006. 7

[6] C. Erdem, B. Sankur, and A. Tekalp. Performance measures for video object segmentation and tracking. IEEE Transactions on Image Processing, 13, 2004. 1

[7] Y. Gal and Z. Ghahramani. Dropout as a bayesian approximation: Representing model uncertainty in deep learning. In Proceedings of the 33rd International Conference on International Conference on Machine Learning - Volume 48, ICML'16, pages 1050-1059. JMLR.org, 2016. 1

[8] D. Giordano, I. Kavasidis, S. Palazzo, and C. Spampinato. Rejecting false positives in video object segmentation. In G. Azzopardi and N. Petkov, editors, Computer Analysis of Images and Patterns, pages 100-112, Cham, 2015. Springer International Publishing. 1

[9] D. Hendrycks and K. Gimpel. A baseline for detecting misclassified and out-of-distribution examples in neural networks. CoRR, abs/1610.02136, 2016. 1

[10] P.-Y. Huang, W.-T. Hsu, C.-Y. Chiu, T.-F. Wu, and M. Sun. Efficient uncertainty estimation for semantic segmentation in videos. In European Conference on Computer Vision (ECCV), 2018. 1

[11] P. Jaccard. The distribution of the flora in the alpine zone. New Phytologist, 11(2):37-50, Feb. 1912. 1

[12] M. Kampffmeyer, A.-B. Salberg, and R. Jenssen. Semantic segmentation of small objects and modeling of uncertainty in urban remote sensing images using deep convolutional neural networks. 2016 IEEE Conference on Computer Vision and Pattern Recognition Workshops (CVPRW), pages 680688, 2016. 1

[13] A. Kendall, V. Badrinarayanan, and R. Cipolla. Bayesian segnet: Model uncertainty in deep convolutional encoderdecoder architectures for scene understanding. CoRR, abs/1511.02680, 2015. 1

[14] S. Liang, Y. Li, and R. Srikant. Principled detection of out-of-distribution examples in neural networks. CoRR, abs/1706.02690, 2017. 1
[15] P. Oberdiek, M. Rottmann, and H. Gottschalk. Classification uncertainty of deep neural networks based on gradient information. In Artificial Neural networks and Pattern Recognition (ANNPR), 2018. 1

[16] M. Rottmann, P. Colling, T. Hack, F. Hüger, P. Schlicht, and H. Gottschalk. Prediction error meta classification in semantic segmentation: Detection via aggregated dispersion measures of softmax probabilities. CoRR, abs/1811.00648, 2018. $1,2,4,5,7,8$

[17] M. Sandler, A. G. Howard, M. Zhu, A. Zhmoginov, and L.-C. Chen. Inverted residuals and linear bottlenecks: Mobile networks for classification, detection and segmentation. CoRR, abs/1801.04381, 2018. 2, 5

[18] C. E. Shannon. A Mathematical Theory of Communication, volume 27, pages 379-423, 623-656. Nokia Bell Labs, 1948. 3

[19] K. Wickstrøm, M. Kampffmeyer, and R. Jenssen. Uncertainty and interpretability in convolutional neural networks for semantic segmentation of colorectal polyps. CoRR, abs/1807.10584, 2018. 1 